\title{
MATHEMATICAL MODELLING OF PLASMA NITRIDING OF AUSTENITIC STAINLESS STEEL
}

\author{
L. F. Spevak ${ }^{1}$, O. A. Nefedova ${ }^{1}$, A. V. Makarov ${ }^{1,2}$, G. V. Samoilova ${ }^{2}$ \\ ${ }^{1}$ Institute of Engineering Science, Ural Branch of the Russian Academy of Sciences, 34 Komsomolskaya st., \\ Ekaterinburg, Russian Federation \\ ${ }^{2}$ M.N. Miheev Institute of Metal Physics of Ural Branch of Russian Academy of Sciences, 18 S. Kovalevskoy st., \\ Ekaterinburg, Russian Federation
}

*Corresponding author. E-mail: nefedova@imach.uran.ru; address for correspondence: ul. Komsomolskaya, 34, Ekaterinburg, Russian Federation. Tel.: +7 (343) 37535 92; fax: 3745330

Two mathematical models of diffusion are discussed as applied to the description of ion nitriding of austenitic stainless steel in electron beam plasma. One model is based on the assumption of the diffusion coefficient dependent on concentration, and this corresponds to the nonlinear boundary value problem of diffusion. The other model takes into account the effect of internal stresses, occurring in the surface layer and induced by introduced nitrogen atoms, on the diffusion process, and this leads to the inhomogeneous boundary value problems of diffusion. Algorithms for solving the boundary value problems are proposed, which are based on the boundary element method. Model examples have been solved to illustrate the functioning of the algorithms. method.

Keywords: mathematical modelling, nitrogen diffusion, plasma nitriding, boundary element

DOI: $10.17804 / 2410-9908.2015 .6 .068-079$

\section{References}

1. Berlin E.V., Koval N.N., Seidman L.A. Plazmennaya khimiko-termicheskaya obrabotka poverkhnosti stalnykh detalei [Plasma Thermochemical Treatment of Steel Part Surfaces]. Moscow, Tekhnosfera Publ., 2012, 464 p. (In Russian).

2. Gavrilov N.V., Mamaev A.S. A Method for Plasma Nitriding of a Steel or Non-Ferrous Product. Russ. Federation Patent 2009100619/02. (In Russian).

3. Gavrilov N.V., Men'shakov A.I. A source of broad electron beams with a self-heated hollow cathode for plasma nitriding of stainless steel. Instruments and Experimental Techniques, 2011, vol. 54, no. 5, pp. 732-739. DOI: 10.1134/S0020441211050046.

4. Gavrilov N.V., Menshakov A.I. Low-temperature nitriding of stainless steel in electron beam plasma at $400^{\circ} \mathrm{C}$. Fizika i khimiya obrabotki materialov, 2012. no. 5, pp. 31-36. (In Russian). 5. Lo K.H., Shek C.H., Lai J.K.L. Recent developments in stainless steels. Materials Science and Engineering: $R$ : Reports, 2009, vol. 65, iss. 4-6, pp. 39-104. DOI: 10.1016/j.mser.2009.03.001.

6. Laleh M., Kargar F., Velashjerdi M. Low-Temperature Nitriding of Nanocrystalline Stainless Steel and Its Effect on Improving Wear and Corrosion Resistance. Journal of Material Engineering and Performance, 2013, vol. 22, iss. 5, pp. 1304-1310. DOI: 10.1007/s11665-012-0417-7.

7. Makarov A.V., Skorynina P.A., Osintseva A.L., Yurovskikh A.S., Savray R.A. Improving the tribological properties of austenitic steel $12 \mathrm{Kh} 18 \mathrm{~N} 10 \mathrm{~T}$ by nanostructuring frictional treatment. Obrabotka metallov: tekhnologiya, oborudovanie, instrumenty, 2015, vol. 69, no. 4, pp. 80-92. (In Russian).

8. Tikhonov A.N., Samarsky A.A. Uravneniya matematicheskoy fiziki [Equations of Mathematical Physics]. M., Moscow University Publ., 1999, 799 p. (In Russian).

9. Brebbia C.A., Telles J.F.C., Wrobel L.C. Boundary Element Techniques. Berlin, Neidelberg, New-York, Tokyo, Springer-Verlag, 1984, 466 p. DOI: 10.1007/978-3-642-48860-3. 
10. Fedotov V.P., Spevak L.F. Modifitsirovannyi metod granichnykh elementov $v$ zadachakh mekhaniki, teploprovodnosti i diffuzii [A Modified Boundary Element Method in Problems of Mechanics, Heat Conduction and Diffusion]. Ekaterinburg, UrO RAN Publ., 2009, 164 p. (In Russian). 11. Christiansen T., Somers M.A.J. Avoiding ghost stress on reconstruction of stress- and composition-depth profiles from destructive X-ray diffraction depth profiling. Materials Science and Engineering: A, 2006, vol. 424, iss. 1-2, pp. 181-189. DOI: 10.1016/j.msea.2006.03.007.

12. Galdikas A., Moskalioviene T. Stress induced nitrogen diffusion during nitriding of austenitic stainless steel. Computational Materials Science, 2010, vol. 50, iss. 2, pp. 796-799. DOI: 10.1016/j.commatsci.2010.10.018.

13. Galdikas A., Moskalioviene T. Modeling of stress induced nitrogen diffusion in nitrided stainless steel. Surface \& Coatings Technology, 2011, vol. 205, iss. 12, pp. 3742-3746. DOI: 10.1016/j.surfcoat.2011.01.040.

14. Moskalioviene T., Galdikas A. Stress induced and concentration dependent diffusion of nitrogen in plasma nitrided austenitic stainless steel. Vacuum, 2012, vol. 86, iss. 10, pp. 1552-1557. DOI: $10.1016 /$ j.vacuum.2012.03.026.

15. Moskalioviene T., Galdikas A., Rivière J.P., Pichon L. Modeling of nitrogen penetration in polycrystalline AISI 316L austenitic stainless steel during plasma nitriding. Surface \& Coatings Technology, 2011, vol. 205, iss. 10, pp. 3301-3306. DOI: 10.1016/j.surfcoat.2010.11.060.

16. Christiansen T.L., Somers M.A.J. Stress and Composition of Carbon Stabilized Expanded Austenite on Stainless Steel. Metallurgical and Materials Transactions A, 2009, vol. 40, iss. 8, pp. 1791-1798. DOI: 10.1007/s11661-008-9717-9.

17. Christiansen T.L., Somers M.A.J. The Influence of Stress on Interstitial Diffusion - Carbon Diffusion Data in Austenite Revisited. Defect and Diffusion Forum, 2010, vol. 297-301, pp. 1408-1413. DOI: 10.4028/www.scientific.net/DDF.297-301.1408.

18. Fedorov A.A. Diffuziya azota v nerzhaveyushchei stali. Tekhnicheskie nauki v Rossii $i$ za rubezhom. In: Technical Sciences in Russia and Abroad. Proceedings of the Third International Scientific Conference. Moscow, Buki-Vedi Publ., 2014, pp. 85-88. (In Russian).

19. Einstein A. Sobranie nauchnykh trudov v chetyrekh tomakh. T. III. I.E. Tamm, Ya.A. Smorodinsky, B.G. Kuznetsov, ed. Raboty po kineticheskoy teorii, teorii izlucheniya $i$ osnovam kvantovoi mekhaniki 1901-1955 [A Collection of Scientific Studies. Vol. III. I.E. Tamm, Ya.A. Smorodinsky, B.G. Kuznetsov, ed. Works on Kinetic Theory, Radiation Theory and Foundations of Quantum Mechanics]. M., Nauka Publ., 1966, pp. 108-117. (In Russian).

20. Kazakov A.L., Spevak L.F. The boundary element method and the power series method in one-dimensional problems of nonlinear filtering. Izv. Irkutskogo gos. un-ta. Ser. Matematika, 2012, vol. 5, no. 2, pp. 2-17. (In Russian).

21. Kazakov A.L., Spevak L.F. Numerical and analytical studies of a nonlinear parabolic equation with boundary conditions of a special form. Applied Mathematical Modelling, 2013, vol. 37, iss. 10-11, pp. 6918-6928. DOI: 10.1016/j.apm.2013.02.026. 
Подана в журнал: 23.11.2015 г.

УДК 533.6:519.6

DOI: $10.17804 / 2410-9908.2015 .6 .068-079$

\title{
МАТЕМАТИЧЕСКОЕ МОДЕЛИРОВАНИЕ ПЛАЗМЕННОГО АЗОТИРОВАНИЯ АУСТЕНИТНОЙ НЕРЖАВЕЮЩЕЙ СТАЛИ
}

\author{
Л. Ф. Спевак ${ }^{1}$, О. А. Нефедова ${ }^{1}$, А. В. Макаров ${ }^{1,2}$, Г. В. Самойлова ${ }^{2}$ \\ ${ }^{1}$ Федеральное государственное бюджетное учреждение науки Институт физики металлов имени \\ М.Н. Михеева Уральского отделения Российской академии наук, 620990, ул. С.Ковалевской, 18 , \\ Екатеринбург, Российская Федерачия \\ ${ }^{2}$ Федеральное государственное бюджетное учреждение науки Институт физики металлов имени \\ М.Н. Михеева Уральского отделения Российской академии наук, 620990, ул. С.Ковалевской, 18, \\ Екатеринбург, Российская Федерачия
}

\begin{abstract}
*Ответственный автор. Электронная почта: nefedova@imach.uran.ru; адрес для переписки: 620049 , ул. Комсомольская, 34, Екатеринбург, Российская Федерация. Телефон: +7 (343) 375-35-92; факс: +7 (343) 374-53-30
\end{abstract}

Рассматриваются две математические модели процесса диффузии применительно к описанию ионного азотирования аустенитной нержавеющей стали в плазме электронного пучка. В основе первой модели лежит предположение о зависимости коэффициента диффузии от концентрации, что соответствует нелинейной краевой задаче диффузии. Вторая модель учитывает влияние на диффузионный процесс внутренних напряжений, возникающих в поверхностном слое и индуцированных внедренными атомами азота, что приводит к последовательности неоднородных краевых задач диффузии. Предложены алгоритмы решения краевых задач, основанные на методе граничных элементов. Решены модельные примеры, иллюстрирующие работу алгоритмов.

Ключевые слова: математическое моделирование, диффузия азота, плазменное азотирование, метод граничных элементов.

\section{1. Введение}

Широкое применение во многих отраслях промышленности (нефтегазовой, химической, пищевой и др.) находят аустенитные хромоникелевые стали, обладающие высокой коррозионной стойкостью и технологичностью. Хотя эти стали обладают низкими прочностными свойствами и склонностью к адгезии при трении, которые не улучшаются термической обработкой. Кардинальное повышение функциональных характеристик хромоникелевых аустенитных сталей может быть достигнуто модифицированием их поверхностного слоя атомами азота путем проведения низкотемпературного ионно-плазменного азотирования [1]. Перспективным методом ионно-плазменного азотирования является азотирование в плазме электронного пучка [2-4].

Снижение температуры азотирования нержавеющих сталей ниже $450{ }^{\circ} \mathrm{C}$ позволяет формировать высокопрочную (твердостью 12-15 ГПа) коррозионностойкую фазу пересыщенного азотом твердого раствора. При низкотемпературном плазменном азотировании удается избежать нежелательного образования нитридов хрома, при котором снижается поток атомов хрома к поверхности, что приводит к потере способности формировать антикоррозийный защитный оксидный слой $[5,6]$. Скорость диффузии азота в материал зависит от энергии активации диффузии и уменьшается с понижением температуры азотирования. Коэффициент диффузии азота и, соответственно, скорость азотирования и глубина азотированных слоев могут быть повышены проведением перед азотированием деформационного наноструктурирования поверхностного слоя [6]. В качестве наноструктурирующей обработки по- 
верхности аустенитных нержавеющих сталей перспективно применение фрикционной обработки скользящим индентором [7].

Все больший интерес вызывают вопросы математического моделирования процессов диффузии атомов внедрения (азот, углерод) в кристаллическую решетку сталей и сплавов. В основе этих моделей лежит дифференциальное уравнение параболического типа - уравнение диффузии. Теория решения линейных задач для уравнений математической физики разработана довольно хорошо [8-10], однако в нелинейном случае, наиболее интересном как с теоретической точки зрения, так и для приложений, имеются только отдельные научные результаты. А моделирование таких процессов, как формирование упрочненного поверхностного слоя при азотировании, приводит к нелинейным задачам, требующим разработки специальных методов решения. В литературе приводятся примеры моделирования этого процесса, однако они используют для оценки напряжений известные решения задач для гомогенных сред [11]. В работах [12-14] предложены модели процесса азотирования, учитывающие усиление диффузии за счет внутренних напряжений, возникающих в поверхностном слое с высокой концентрацией внедренных атомов азота в крупнокристаллическую аустенитную сталь, которые, однако, не учитывают изменения напряженного состояния в градиентном поверхностном слое при деформационном наноструктурировании. Делаются попытки учета при моделировании низкотемпературного плазменного азотирования анизотропии коэффициентов диффузии для различных кристаллографических ориентировок аустенитной стали [15]. В работах [16, 17] предложены модели процесса диффузии углерода, но физическое влияние азота несколько отличается и напрямую эти результаты использовать затруднительно.

Таким образом, актуальной и востребованной задачей является проведение теоретического исследования процессов низкотемпературного азотирования аустенитных сталей с использованием современных подходов к решению краевых задач математической физики. В дальнейшем проведение совместного теоретического и прямого экспериментального определения концентраций азота по глубине азотированных слоев позволит решать обратные задачи диффузии с целью корректировки моделей, включая определение коэффициентов диффузии в недеформированных и наноструктурированных поверхностных слоях, а также граничных условий для краевых задач. Это важно в связи с существенной неравномерностью распределения примеси азота в пограничном слое, что приводит к большим градиентам изменения физических и механических свойств слоя. Представляется, что только совместное использование экспериментальных методов и методов физического и математического моделирования позволит корректно решать задачу оптимизации химического и структурнофазового состава градиентного поверхностного слоя для заданных условий эксплуатации.

Цель настоящей работы заключается в обзоре постановок краевой задачи диффузии, описывающей процесс плазменного азотирования, и разработке алгоритмов решения этой задачи. На основе предложенных алгоритмов в дальнейшем будет создана компьютерная программа, позволяющая путем сравнения теоретических и экспериментально полученных распределений концентраций азота по глубине азотированных слоев решать обратные задачи диффузии для корректировки математической модели и для определения коэффициента диффузии.

\section{2. Математическая постановка задачи}

\section{1 Нелинейное уравнение диффузии}

Процесс диффузии газа описывается дифференциальным уравнением диффузии вторым законом Фика. В работе рассматривается одномерный диффузионный процесс в области $x \in[0, h]$, где $h$ - толщина плоского образца, граница $x=0$ соответствует поверхности, контактирующей с азотом. Нелинейность процесса диффузии определяется зависимостью коэффициента диффузии от концентрации. Уравнение диффузии в этом случае имеет вид: 


$$
\frac{\partial c(x, t)}{\partial t}=\frac{\partial}{\partial x}\left(D(c) \frac{\partial c(x, t)}{\partial x}\right)
$$

при заданных начальных и граничных условиях. Здесь $c(x, t)$ - распределение концентрации азота в образце; $t$ - время азотирования.

Предполагается, что в начальный момент времени $t=0$ азота в образце не было, начальное условие задается в виде:

$$
\left.c(x, t)\right|_{t=0}=0 .
$$

Так как при ионном азотировании аустенитной нержавеющей стали в плазме электронного пучка происходит диффузия азота вглубь образца [18], то можно считать концентрацию азота на поверхности постоянной

$$
\left.c(x, t)\right|_{x=0}=c^{*}(t),
$$

полагая, что равновесная с окружающей средой концентрация азота на поверхности достигается за пренебрежимо малое время.

Альтернативным граничным условием при $x=0$ является задание диффузионного потока:

$$
\left.f(x, t)\right|_{x=0}=f^{*}(t)
$$

Как правило, толщина диффузионного слоя при азотировании много меньше соответствующего размера образца, поэтому рассматриваемая задача подобна диффузии в полуограниченной области, когда выполняется условие $\left.f(x, t)\right|_{x \rightarrow \infty}=0$. В связи с этим на удаленной границе принимается условие

$$
\left.f(x, t)\right|_{x=h}=0
$$

В качестве вида зависимости коэффициента диффузии от концентрации при плазменном азотировании аустенитной нержавеющей стали в работах [12 - 14] предложено использовать широко известное соотношение Эйнштейна-Смолуховского $D(c)=F(1 / c)[19]$ в виде

$$
D(c)=\frac{\alpha}{c} ; \alpha=R T L
$$

где $R$ - универсальная газовая постоянная; $T$ - абсолютная температура; $L$ - коэффициент Онзагера. Подстановка (5) в уравнении (1) приводит уравнение диффузии к виду

$$
\frac{\partial c(x, t)}{\partial t}=\frac{\alpha}{c} \frac{\partial^{2} c(x, t)}{\partial x^{2}}-\frac{\alpha}{c^{2}}\left(\frac{\partial c(x, t)}{\partial x}\right)^{2} .
$$

\section{2 Модель, учитывающая усиление диффузии за счет градиента внутренних напряэений}


Насыщение стали азотом в процессе плазменного азотирования приводит к увеличению ее удельного объема. Кроме того, вблизи поверхности образца происходит образование и взаимодействие различных дефектов кристаллической решетки, накопление атомов азота, образование новых фаз и интенсивное ионное распыление. Совокупность этих эффектов приводит к возникновению сжимающих внутренних напряжений в азотированном слое.

Вторая модель рассматривает диффузию с учетом влияния внутренних напряжений, вызванных внедренными атомами азота. Известно, что внутренние напряжения оказывают влияние на диффузию азота в соответствии со следующим уравнением:

$$
f(x, t)=-D \frac{\partial c(x, t)}{\partial x}+\frac{D V_{N}}{R T} c(x, t) \frac{\partial \sigma(x, t)}{\partial x} .
$$

Здесь $V_{N}$ - парциальный молярный объем азота в стали; $\sigma(x, t)$ - внутренние напряжения. Уравнение (7) и закон сохранения вещества

$$
\frac{\partial f(x, t)}{\partial x}=-\frac{\partial c(x, t)}{\partial t}
$$

приводят к уравнению для концентрации

$$
\frac{\partial c(x, t)}{\partial t}=D \frac{\partial^{2} c(x, t)}{\partial x^{2}}-\frac{D V_{N}}{R T}\left(\frac{\partial c(x, t)}{\partial x} \frac{\partial \sigma(x, t)}{\partial x}+c(x, t) \frac{\partial^{2} \sigma(x, t)}{\partial x^{2}}\right)
$$

В силу малости множителя $\frac{\partial^{2} \sigma(x, t)}{\partial x^{2}}$ вторым слагаемым, стоящим в скобках в правой части уравнения (9), можно пренебречь, тогда

$$
\frac{\partial c(x, t)}{\partial t}=D \frac{\partial^{2} c(x, t)}{\partial x^{2}}-\frac{D V_{N}}{R T} \frac{\partial c(x, t)}{\partial x} \frac{\partial \sigma(x, t)}{\partial x} .
$$

В работах [12 - 14] предложено при азотировании аустенитной нержавеющей стали использовать линейную зависимость внутренних напряжений от концентрации в виде:

$$
\sigma(x, t)=-X \cdot c(x, t)
$$

где коэффициент пропорциональности принимается равным $X=200 \frac{M \Pi a}{a m . \%}$. После подстановки зависимости (11) в (10) получаем

$$
\frac{\partial c(x, t)}{\partial t}=D \frac{\partial^{2} c(x, t)}{\partial x^{2}}+X \frac{D V_{N}}{R T}\left(\frac{\partial c(x, t)}{\partial x}\right)^{2}
$$

Дополняя уравнение (12) краевыми условиями (2) - (4), получаем еще одну краевую задачу, описывающую процесс диффузии. 
open-access journal

\section{3. Алгоритмы решения методом граничных элементов}

\section{1 Алгоритм решения линейного однородного уравнения}

Краевая задача (1), (2), (3), (4) в случае постоянного коэффициента диффузии имеет известное решение методом граничных элементов $[9,10]$. В момент наблюдения $t_{F}$ в произвольной точке $\xi \in[0, h]$ справедливо равенство

$$
c\left(\xi, t_{F}\right)=D \int_{0}^{t_{F}}\left[q(0, t) u^{*}\left(\xi, 0, t_{F}, t\right)-c^{*} q^{*}\left(\xi, 0, t_{F}, t\right)-c(h, t) q^{*}\left(\xi, h, t_{F}, t\right)\right] d t .
$$

Здесь $t_{F}>0 ; q(x, t)=\frac{\partial c(x, t)}{\partial n}-$ производная концентрации по внешней нормали к границе, в граничной точке с внешней нормалью $n(x)$ величина $q(x, t)=-\frac{f(x, t)}{D} n(x) ; n(0)=-1$, $n(h)=1 ; u^{*}\left(\xi, x, t_{F}, t\right)=\frac{1}{\sqrt{4 \pi k\left(t_{F}-t\right)}} \exp \left(-\frac{(\xi-x)^{2}}{4 k\left(t_{F}-t\right)}\right)$
$q^{*}\left(\xi, x, t_{F}, t\right)=-\frac{(x-\xi) n(x)}{4 \sqrt{\pi}\left(k\left(t_{F}-t\right)\right)^{\frac{3}{2}}} \exp \left(-\frac{(\xi-x)^{2}}{4 k\left(t_{F}-t\right)}\right)$

Граничные интегральные уравнения имеют вид:

$$
\begin{gathered}
\frac{1}{2} c^{*}=D \int_{0}^{t_{F}}\left[q(0, t) u^{*}\left(0,0, t_{F}, t\right)-c^{*}(t) q^{*}\left(0,0, t_{F}, t\right)-c(h, t) q^{*}\left(0, h, t_{F}, t\right)\right] d t \\
\frac{1}{2} c(h, t)=D \int_{0}^{t_{F}}\left[q(0, t) u^{*}\left(h, 0, t_{F}, t\right)-c^{*}(t) q^{*}\left(h, 0, t_{F}, t\right)-c(h, t) q^{*}\left(h, h, t_{F}, t\right)\right] d t .
\end{gathered}
$$

Рассмотрим процесс диффузии на интервале $t \in\left[0, t_{M}\right]$, разбитом точками $t_{0}=0, t_{1}, t_{2}, \ldots, t_{M}$ на $M$ шагов. На интервале $t \in\left[t_{n-1}, t_{n}\right]$, считаем постоянными $q(0, t)=q_{0}^{(n)}$, $c(0, t)=c^{*(n)}, c(h, t)=c_{h}^{(n)}$. Тогда в момент наблюдения $t_{n}$ граничные интегральные уравнения имеют вид:

$$
\begin{aligned}
& \frac{1}{2} c^{*(n)}=D\left[\sum_{i=1}^{n}\left(q_{0}^{(i)} \int_{t_{i-1}}^{t_{i}} u^{*}\left(0,0, t_{n}, t\right) d t-c^{*(i)} \int_{t_{i-1}}^{t_{i}} q^{*}\left(0,0, t_{n}, t\right) d t-c_{h}^{(i)} \int_{t_{i-1}}^{t_{i}} q^{*}\left(0, h, t_{n}, t\right) d t\right)\right] ; \\
& \frac{1}{2} c_{h}^{(n)}=D\left[\sum_{i=1}^{n}\left(q_{0}^{(i)} \int_{t_{i-1}}^{t_{i}} u^{*}\left(h, 0, t_{n}, t\right) d t-c^{*(i)} \int_{t_{i-1}}^{t_{i}} q^{*}\left(h, 0, t_{n}, t\right) d t-c_{h}^{(i)} \int_{t_{i-1}}^{t_{i}} q^{*}\left(h, h, t_{n}, t\right) d t\right)\right] .
\end{aligned}
$$

Здесь $q_{0}^{(i)}, c_{h}^{(i)}, i=1,2, \ldots, n-1$, найдены на предыдущих шагах; $q_{0}^{(n)}, c_{h}^{(n)}$ - неизвестные, определяемые из решения системы (16), (17). Тогда решение краевой задачи (1)-(4) в момент времени $t=t_{n}$ определяется по формуле: 
open-recess journal

$$
c\left(\xi, t_{n}\right)=D\left[\sum_{i=1}^{n}\left(q_{0}^{(i)} \int_{t_{i-1}}^{t_{i}} u^{*}\left(\xi, 0, t_{n}, t\right) d t-c_{h}^{(i)} \int_{t_{i-1}}^{t_{i}} q^{*}\left(\xi, h, t_{n}, t\right) d t\right)-c^{*} \int_{0}^{t_{n}} q^{*}\left(\xi, 0, t_{n}, t\right) d t\right] .
$$

В результате на каждом шаге по времени получаем непрерывное по пространственной координате решение вида (18). Решение задачи в случае граничного условия (3') проводится аналогично.

В случае, когда граничное условие (3) (или (3')) не зависит от времени, $c^{*}=$ const $\left(f^{*}=\right.$ const $)$, соотношения (13)-(15) приводят к известным аналитическим решениям соответственно

$$
c(\xi, t)=c^{*}\left(1-\operatorname{erf}\left(\frac{\xi}{2 \sqrt{D t}}\right)\right)
$$

или

$$
c(\xi, t)=\frac{2 f^{*} \sqrt{t}}{\sqrt{D}}\left(\frac{1}{\sqrt{\pi}} \exp \left(-\frac{\xi^{2}}{4 D t}\right)-\frac{\xi}{2 \sqrt{D t}} \operatorname{erf}\left(\frac{\xi}{2 \sqrt{D t}}\right)\right)
$$

где $\operatorname{erf}(y)=\left(\frac{2}{\sqrt{\pi}}\right) \int_{0}^{y} \exp \left(-z^{2}\right) d z-$ функция ошибок.

\section{2 Алгоритмы решения нелинейного уравнения диффузии}

В случае, когда коэффициент диффузии зависит от концентрации, уравнение диффузии становится нелинейным, как, например, уравнение (6). Тогда применение алгоритма, описанного в предыдущем разделе, может оказаться невозможным. В этом случае на каждом шаге по времени исходное уравнение (1) может быть представлено в виде уравнения Пуассона

$$
\frac{\partial^{2} c(x, t)}{\partial x^{2}}=\frac{1}{D(c)}\left(\frac{\partial c(x, t)}{\partial t}-\frac{\partial D(c)}{\partial c}\left(\frac{\partial c(x, t)}{\partial x}\right)\right)
$$

которое при граничных условиях (3) (или (3’)), (4) решается итерационно на основе подхода, предложенного в работах [20, 21]. При условиях (3), (4) $k$-я итерация имеет вид:

$$
\begin{gathered}
c^{(k)}(\xi)=q_{1}^{(k)} \bar{u}^{*}(\xi, 0)-c^{*} \bar{q}^{*}(\xi, 0)-c_{2}^{(k)} \bar{q}^{*}(\xi, h)- \\
-\int_{0}^{h} \frac{1}{D\left(c^{(k-1)}\right)}\left(\frac{\partial c^{(k-1)}(x, t)}{\partial t}-\frac{\partial D\left(c^{(k-1)}\right)}{\partial c}\left(\frac{\partial c^{(k-1)}(x, t)}{\partial x}\right)\right) \bar{u}^{*}(\xi, x) d x
\end{gathered}
$$

где $\xi \in(0, h) ; c_{2}=\left.c\right|_{x=h}, q_{1}=\left.q\right|_{x=0} ; \bar{u}^{*}(\xi, x)=\frac{1}{2}(h-|r|) ; \bar{q}^{*}(\xi, x)=-\frac{1}{2} \operatorname{sgn}(r) n, r=x-\xi$. Heизвестные величины $q_{1}^{(k)}$ и $c_{2}^{(k)}$ определяются на каждой итерации из решения граничных интегральных уравнений 


$$
\left\{\begin{array}{l}
\frac{1}{2} c^{*}=\frac{h}{2} q_{1}+\frac{1}{2} c_{2}-\int_{0}^{h} \frac{1}{D\left(c^{(k-1)}\right)}\left(\frac{\partial c^{(k-1)}(x, t)}{\partial t}-\frac{\partial D\left(c^{(k-1)}\right)}{\partial c}\left(\frac{\partial c^{(k-1)}(x, t)}{\partial x}\right)\right) \bar{u}^{*}(0, x) d x, \\
\frac{1}{2} c_{2}=\frac{1}{2} c^{*}-\int_{0}^{h} \frac{1}{D\left(c^{(k-1)}\right)}\left(\frac{\partial c^{(k-1)}(x, t)}{\partial t}-\frac{\partial D\left(c^{(k-1)}\right)}{\partial c}\left(\frac{\partial c^{(k-1)}(x, t)}{\partial x}\right)\right) \bar{u}^{*}(h, x) d x .
\end{array}\right.
$$

Итерационный процесс закачивается, когда выполнено условие близости двух последовательных итераций. В результате мы вновь на каждом шаге получим распределение концентрации в образце в непрерывном виде.

Уравнение (12), учитывающее влияние градиента внутренних напряжений, также является нелинейным. Однако линейность слагаемого, содержащего старшую производную, позволяет решать это уравнение при краевых условиях (2)-(4) аналогично задаче (1)-(4). При этом нелинейная задача сводится к последовательности решения линейных неоднородных задач. На каждом шаге по времени это вновь приводит к итерационному процессу. На $k$-й итерации граничные интегральные уравнения

$$
\begin{gathered}
\frac{1}{2} c^{*(n)}=D\left[\sum_{i=1}^{n}\left(q_{0}^{(i)} \int_{t_{i-1}}^{t_{i}} u^{*}\left(0,0, t_{n}, t\right) d t-c^{*(i)} \int_{t_{i-1}}^{t_{i}} q^{*}\left(0,0, t_{n}, t\right) d t-c_{h}^{(i)} \int_{t_{i-1}}^{t_{i}} q^{*}\left(0, h, t_{n}, t\right) d t\right)\right]+ \\
+X \frac{D V_{N}}{R T} \int_{0}^{t_{n} h} \int_{0}^{h} \frac{\partial c^{(k-1)}(x, t)}{\partial x} u^{*}\left(0, x, t_{n}, t\right) d x d t \\
\frac{1}{2} c_{h}^{(n)}=D\left[\sum_{i=1}^{n}\left(q_{0}^{(i)} \int_{t_{i-1}}^{t_{i}} u^{*}\left(h, 0, t_{n}, t\right) d t-c^{*(i)} \int_{t_{i-1}}^{t_{i}} q^{*}\left(h, 0, t_{n}, t\right) d t-c_{h}^{(i)} \int_{t_{i-1}}^{t_{i}} q^{*}\left(h, h, t_{n}, t\right) d t\right)\right]+ \\
+X \frac{D V_{N}}{R T} \int_{0}^{t_{n}} \int_{0}^{h} \frac{\partial c^{(k-1)}(x, t)}{\partial x} u^{*}\left(h, x, t_{n}, t\right) d x d t
\end{gathered}
$$

определят неизвестные $q_{0}^{(n)}, c_{h}^{(n)}$ на данной итерации. Тогда $k$-я итерация решения краевой задачи в момент времени $t=t_{n}$ имеет вид:

$$
\begin{gathered}
c^{(k)}\left(\xi, t_{n}\right)=D\left[\sum_{i=1}^{n}\left(q_{0}^{(i)} \int_{t_{i-1}}^{t_{i}} u^{*}\left(\xi, 0, t_{n}, t\right) d t-c_{h}^{(i)} \int_{t_{i-1}}^{t_{i}} q^{*}\left(\xi, h, t_{n}, t\right) d t\right)-c^{*} \int_{0}^{t_{n}} q^{*}\left(\xi, 0, t_{n}, t\right) d t\right]+ \\
+X \frac{D V_{N}}{R T} \int_{0}^{t_{n} h} \frac{\partial c^{(k-1)}(x, t)}{\partial x} u^{*}\left(\xi, x, t_{n}, t\right) d x d t
\end{gathered}
$$

\section{4. Примеры}

Предложенные алгоритмы были применены для решения модельных задач при следующих значениях параметров задач. Алгоритм (13)-(18) при постоянном коэффициенте диффузии $D=6 \cdot 10^{-11} \frac{\mathrm{cm}^{2}}{c}$ и постоянном значении $f^{*}=-1,8 \cdot 10^{-8} \frac{1}{c \cdot \mathrm{cm}^{2}}$, соответствующем 
opter-access journal

$q(0, t)=-\frac{f^{*}}{D}=300 \frac{1}{c M^{4}}$, привел к решению, показанному на рис. 1 и совпадающему с решением (19').

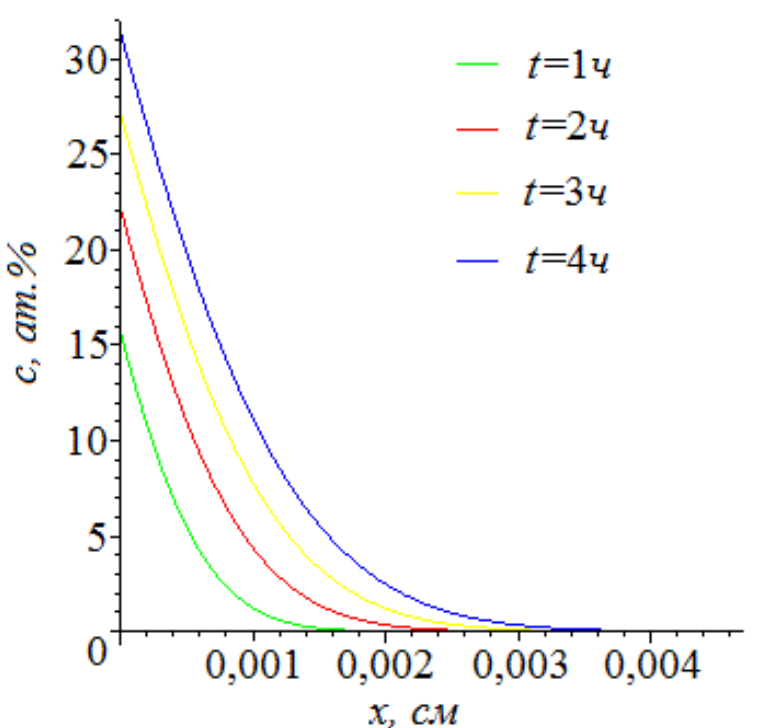

Рис. 1. Концентрация азота при постоянном коэффициенте диффузии при различных периодах азотирования

Решение с помощью алгоритма (23)-(25) неоднородной задачи, учитывающей влияние градиентов напряжений при тех же граничных условиях и следующих значениях параметров: $X=200 \frac{M \Pi a}{a m . \%}, V_{N}=40$ cм $^{3}, R=8,314 \frac{\text { Дж }}{K \cdot \text { моль }}, T=673 K$, показано на рис. 2.

Сравнение графиков на рис. 1 и 2, соответствующих одинаковым периодам азотирования, показывает ускорение процесса диффузии вследствие влияния внутренних напряжений.

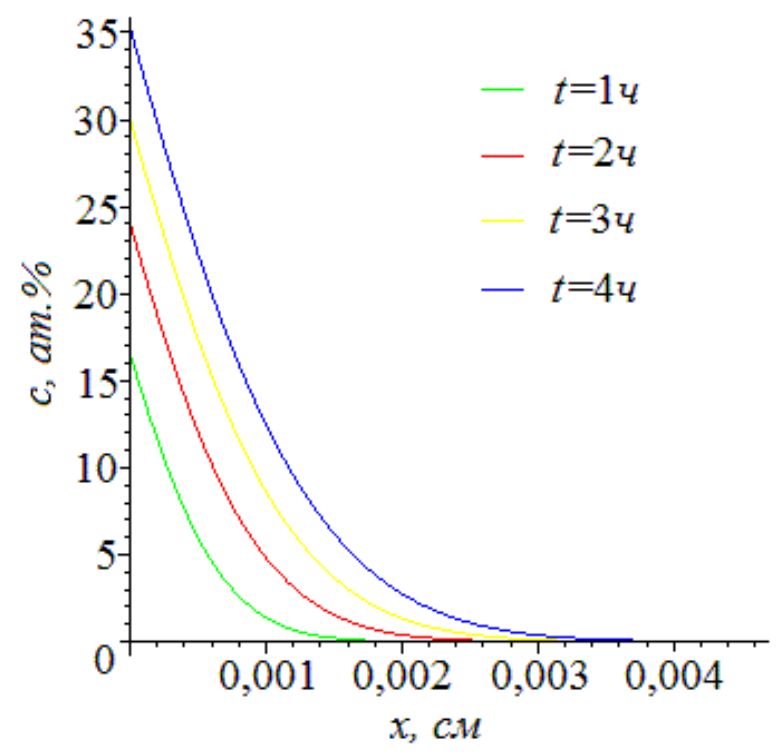

Рис.2. Концентрация азота с учетом внутренних напряжений при различных периодах азотирования

\section{5. Заключение}


Обоснована актуальность построения математических моделей и теоретического изучения процессов диффузии азота при низкотемпературном ионно-плазменном азотировании, в частности, в плазме электронного пучка, нержавеющих аустенитных сталей. Отмечается важность разработки эффективных алгоритмов решения краевых задач диффузии. Предложенные алгоритмы решения линейной и нелинейных краевых задач диффузии на основе метода граничных элементов могут быть использованы для создания компьютерных программ и решения обратной задачи диффузии азота.

\section{Благодарность}

Работа выполнена в рамках государственного задания ФАНО России (тема «Структура» № 01201463331) при поддержке РФФИ (проект № 15-08-07947).

\section{Литература}

1. Берлин Е. В., Коваль Н. Н., Сейдман Л. А. Плазменная химико-термическая обработка поверхности стальных деталей. - М. : Техносфера, 2012. - 464 с. - ISBN 978-5-94836-328-8.

2. Способ плазменного азотирования изделия из стали или из цветного сплава : пат. 2413033 Рос. Федерация / Гаврилов Н. В., Мамаев А. С., Государственное учреждение Институт электрофизики УрО РАН. - № 2009100619/02 ; заявл. 11.01.09 ; опубл. 27.02.11, Бюл. № $6 .-9 \mathrm{c}$.

3. Gavrilov N. V., Men'shakov A. I. A source of broad electron beams with a self-heated hollow cathode for plasma nitriding of stainless steel. Instruments and Experimental Techniques. 2011. -Vol. 54, no. 5. - P. 732-739. - DOI: 10.1134/S0020441211050046.

4. Гаврилов Н. В., Меньшаков А. И. Низкотемпературное азотирование нержавеющей стали в плазме электронного пучка при $400^{\circ} \mathrm{C} / /$ Физика и химия обработки материалов. 2012. - № 5. - C. 31-36.

5. Lo K. H., Shek C. H., Lai J. K. L. Recent developments in stainless steels // Materials Science and Engineering: R: Reports. - 2009. - Vol. 65, iss. 4-6. - P. 39-104. - DOI: 10.1016/j.mser.2009.03.001.

6. Laleh M., Kargar F., Velashjerdi M. Low-temperature Nitriding of Nanocrystalline Stainless Steel and Its Effect on Improving Wear and Corrosion Resistance // Journal of Material Engineering and Performance. - 2013. - Vol. 22, iss. 5. - P. 1304-1310. - DOI: 10.1007/s11665-012-0417-7.

7. Повышение трибологических свойств аустенитной стали 12 Х18Н10Т наноструктурирующей фрикционной обработкой / А. В. Макаров, П. А. Скорынина, А. Л. Осинцева, А. С. Юровских, Р. А. Саврай // Обработка металлов: технология, оборудование, инструменты. - 2015. - T. 69, № 4. - C. 80-92. - DOI: 10.17212/1994-6309-2015-4-80-92.

8. Тихонов А.Н., Самарский А.А. Уравнения математической физики. - М. : Изд-во Моск. ун-та, 1999. - 799 с. - ISBN 5-211-04138-0.

9. Brebbia C. A., Telles J. F. C., Wrobel L. C. Boundary Element Techniques. - Berlin, Neidelberg, New-York, Tokyo : Springer-Verlag, 1984. - 466 p. - ISBN 978-3-642-48862-7. - DOI: 10.1007/978-3-642-48860-3.

10. Федотов В. П., Спевак Л. Ф. Модифицированный метод граничных элементов в задачах механики, теплопроводности и диффузии. - Екатеринбург : УрО РАН, 2009. - 164 с.

11. Christiansen T., Somers M. A. J. Avoiding ghost stress on reconstruction of stress- and composition-depth profiles from destructive X-ray diffraction depth profiling // Materials Science and Engineering: A. - 2006. - Vol. 424, iss 1-2. - P. 181-189. - DOI: 10.1016/j.msea.2006.03.007. 12. Galdikas A., Moskalioviene T. Stress induced nitrogen diffusion during nitriding of austenitic stainless steel // Computational Materials Science. - 2010. - Vol. 50, iss. 2. - P. 796-799. - DOI: 10.1016/j.commatsci.2010.10.018. 
13. Galdikas A., Moskalioviene T. Modeling of stress induced nitrogen diffusion in nitrided stainless steel // Surface \& Coatings Technology. - 2011. - Vol. 205, iss. 12. - P. 3742-3746. DOI: $10.1016 /$ j.surfcoat.2011.01.040.

14. Moskalioviene T., Galdikas A. Stress induced and concentration dependent diffusion of nitrogen in plasma nitrided austenitic stainless steel // Vacuum. - 2012. - Vol. 86, iss 10. - P. 15521557. - DOI: 10.1016/j.vacuum.2012.03.026.

15. Modeling of nitrogen penetration in polycrystalline AISI $316 \mathrm{~L}$ austenitic stainless steel during plasma nitriding / T. Moskalioviene, A. Galdikas, J. P. Rivière, L. Pichon // Surface \& Coatings Technology. - 2011. - Vol. 205, iss. 10. - P. 3301-3306. - DOI: 10.1016/j.surfcoat.2010.11.060.

16. Christiansen T. L., Somers M. A. J. Stress and Composition of Carbon Stabilized Expanded Austenite on Stainless Steel // Metallurgical and Materials Transactions A. - 2009. - V. 40, iss. 8. P. 1791-1798. - DOI: 10.1016/j.surfcoat.2010.11.060.

17. Christiansen T. L., Somers M. A. J. The Influence of Stress on Interstitial Diffusion - Carbon Diffusion Data in Austenite Revisited // Defect and Diffusion Forum. - 2010. - Vol. 297-301. - P. 1408-1413. - DOI: 10.4028/www.scientific.net/DDF.297-301.1408.

18. Федоров А. А. Диффузия азота в нержавеющей стали // Технические науки в России и за рубежом: материалы III междунар. науч. конф. - М. : Буки-Веди, 2014. - С. 85-88. - ISBN 978-5-4465-0465-7.

19. О движении взвешенных в покоящейся жидкости частиц, требуемом молекулярнокинетической теорией теплоты. Гл. 8 // Эйнштейн А. Собрание научных трудов в четырех томах. Том III / Под ред. И. Е. Тамма, Я. А. Смородинского, Б. Г. Кузнецова. - М. : Наука, 1966. - С. 108-117. - ISBN 978-5-458-33554-6.

20. Казаков А. Л., Спевак Л. Ф. Методы граничных элементов и степенных рядов в одномерных задачах нелинейной фильтрации // Изв. Иркутского гос. ун-та. Сер. Математика. 2012. - T. 5, № 2. - C. 2-17.

21. Kazakov A. L., Spevak L. F. Numerical and analytical studies of a nonlinear parabolic equation with boundary conditions of a special form // Applied Mathematical Modelling. - 2013. - Vol. 37, iss. 10-11. - P. 6918-6928. - DOI: 10.1016/j.apm.2013.02.026. 